\title{
The study of the Three Nucleon Force in full QCD Lattice calculations
}

\section{Takumi Doi*}

Graduate School of Pure and Applied Sciences, University of Tsukuba, Tsukuba, Ibaraki

305-8571, Japan

E-mail: doieribf.riken.ip

\section{for HAL QCD Collaboration}

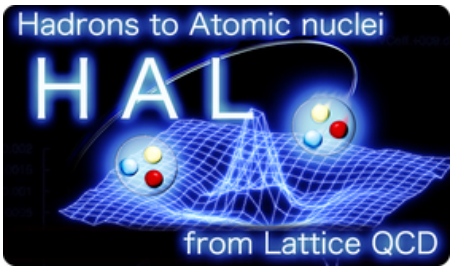

We study the three nucleon force in the triton channel using dynamical clover fermion lattice QCD. The Nambu-Bethe-Salpeter wave function is utilized to obtain the potentials among three nucleons. Since the straightforward calculation is prohibitively expensive, two different frameworks are developed to meet the challenge. In the first method, we study the effective two nucleon potentials in the three nucleon system, where the differences between the effective two nucleon potentials and the genuine two nucleon potentials correspond to the three nucleon system effect, part of which is originated from the three nucleon force. The calculation is performed using $N_{f}=2$ clover fermion at $m_{\pi}=1.13 \mathrm{GeV}$ generated by CP-PACS Collaboration, and $N_{f}=2+1$ clover fermion at $m_{\pi}=0.70,0.57 \mathrm{GeV}$ generated by PACS-CS Collaboration. In the second method, we study the three nucleon system with 3D-configuration of nucleons fixed. This enables us to extract the three nucleon force directly, if both of parity-even and parity-odd two nucleon potentials are provided. Since parity-odd two nucleon potentials are not available in lattice QCD at this moment, we propose a new general procedure to identify the three nucleon force using only parity-even two nucleon potentials. The calculation are performed with $N_{f}=2$ clover fermion at $m_{\pi}=1.13 \mathrm{GeV}$ generated by CP-PACS Collaboration, employing the linear setup for the 3D-configuration. Preliminary results for the scalar/isoscalar three nucleon force are presented.

The XXVIII International Symposium on Lattice Field Theory, Lattice2010

June 14-19, 2010

Villasimius, Italy

\footnotetext{
* Speaker.
} 


\section{Introduction}

Since the celebrated work by Yukawa 75 years ago, the nuclear force has been one of the most essential quantities in the development of nuclear physics, and so-called "realistic nuclear potentials" between two nucleons $(2 \mathrm{~N})$ are available to date. However, recent precise calculations of few-nucleon systems clearly point that the $2 \mathrm{~N}$ force alone is insufficient to understand the nuclei, which calls for three (and/or more) nucleon forces. Actually, the three nucleon force (TNF) is projected to play an important and nontrivial role in various phenomena in nuclear and astro physics. For the binding energies of light nuclei, attractive TNF is required to reproduce the experimental data. On the other hand, repulsive TNF is necessary to reproduce the empirical saturation density of symmetric nuclear matter. For the EoS of asymmetric nuclear matter, repulsive TNF is required to explain the observed maximum neutron star mass. Recently, it is argued that TNF is responsible for the anomaly in the drip line and the nontrivial magic number of neutron-rich nuclei [四].

Although the experimental/theoretical scrutiny of three nucleon scattering are shedding light on the natures of TNF [[]], our knowledge on TNF is still quite limited. Pioneered by FujitaMiyazawa [3], TNF have been mainly studied from the two-pion exchange picture with the $\Delta$ excitation. In addition, repulsive TNF is often introduced phenomenologically [四]. Recently, the TNF based on chiral EFT is developing [5], but the unknown low-energy constants can be obtained only by the fitting to the experimental data. Since TNF is originated by the fact that the nucleon is not a fundamental particle, it is essential to study TNF from the fundamental DoF, i.e., quarks and gluons. In this proceeding, we report such first-principle calculations of TNF using lattice QCD.

In the lattice QCD calculation of nuclear forces, it is recently proposed [ [ $]$ to use the NambuBethe-Salpeter (NBS) wave function so that the potential is faithful to the phase shift by construction. The obtained $2 \mathrm{~N}$ potentials are found to have desirable features, such as attractive well at long and medium distances, and the central repulsive core at short distance [ $[$,, $]$. The method has been successfully extended to the hyperon-nucleon (YN) and hyperon-hyperon (YY) interactions [8, Q]].

We extend this methodology to the three nucleon $(3 \mathrm{~N})$ system, namely, in the triton channel. Due to the significantly enlarged DoF, the straightforward calculation is impossible. We explore two different methods to overcome this problem, one is the study of effective $2 \mathrm{~N}$ potentials in $3 \mathrm{~N}$ system, and the other is the study of $3 \mathrm{~N}$ system with fixed 3D-configuration by the linear setup.

\section{Formulation for the effective $2 \mathrm{~N}$ potential}

Since the detailed formulation to study a $2 \mathrm{~N}$ system is given in Ref. [四], we discuss the extension to a $3 \mathrm{~N}$ system here. We first consider the equal-time NBS wave function $\psi(\vec{r}, \vec{\rho})$, which can be obtained by the calculation of six point correlation function,

$$
G_{\alpha \beta \gamma, \alpha^{\prime} \beta^{\prime} \gamma^{\prime}}\left(\vec{r}, \vec{\rho}, t-t_{0}\right)=\left\langle N_{\alpha}\left(\vec{x}_{1}, t\right) N_{\beta}\left(\vec{x}_{2}, t\right) N_{\gamma}\left(\vec{x}_{3}, t\right) \overline{\left(N_{\alpha^{\prime}}^{\prime}\left(t_{0}\right) N_{\beta^{\prime}}^{\prime}\left(t_{0}\right) N_{\gamma^{\prime}}^{\prime}\left(t_{0}\right)\right)}\right\rangle,
$$

where $\vec{r} \equiv \vec{x}_{1}-\vec{x}_{2}, \vec{\rho} \equiv \vec{x}_{3}-\left(\vec{x}_{1}+\vec{x}_{2}\right) / 2$ are the Jacobi coordinates, and $N$ denotes either of $p$ or $n$.

At the leading order of the velocity expansion of the potentials, the NBS wave function can be converted to the potentials through the following Schrödinger equation,

$$
\left[-\frac{1}{2 \mu_{r}} \nabla_{r}^{2}-\frac{1}{2 \mu_{\rho}} \nabla_{\rho}^{2}+\sum_{i<j} V_{2 N, i j}\left(\vec{r}_{i j}\right)+V_{T N F}(\vec{r}, \vec{\rho})\right] \psi(\vec{r}, \vec{\rho})=E \psi(\vec{r}, \vec{\rho}),
$$


where $V_{2 N, i j}\left(\vec{r}_{i j}\right)$ with $\vec{r}_{i j} \equiv \vec{x}_{i}-\vec{x}_{j}$ denotes the potential between $(i, j)$-pair, $V_{T N F}(\vec{r}, \vec{\rho})$ the TNF, $\mu_{r}=m_{N} / 2, \mu_{\rho}=2 m_{N} / 3$ the reduced masses. If we can calculate $\psi(\vec{r}, \vec{\rho})$ for all $\vec{r}, \vec{\rho}$, and if all $V_{2 N, i j}\left(\vec{r}_{i j}\right)$ are available by (separate) lattice calculations for the genuine $2 \mathrm{~N}$ system, we can extract $V_{T N F}(\vec{r}, \vec{\rho})$. Unfortunately, this is not the case: Since both $\vec{r}$ and $\vec{\rho}$ have $L^{3}$ DoF, the calculation cost is more expensive by a factor of $L^{3}$ compared to the $2 \mathrm{~N}$ system. Furthermore, the number of diagrams to be calculated in the Wick contraction tends to diverge with a factor of $N_{u} ! \times N_{d} !\left(N_{u, d}\right.$ are numbers of $\mathrm{u}, \mathrm{d}$ quarks in the system). We also note that not all $2 \mathrm{~N}$ potentials are available in lattice QCD at this moment: Only parity-even $2 \mathrm{~N}$ potentials have been obtained so far.

In order to avoid these problems, we consider the effective $2 \mathrm{~N}$ potential in the $3 \mathrm{~N}$ system. More specifically, we take the summation over the location of the spectator nucleon $N\left(\vec{x}_{3}\right)$,

$$
\phi(\vec{r}) \equiv \sum_{\vec{x}_{3}} \psi(\vec{r}, \vec{\rho})=\sum_{\vec{\rho}} \psi(\vec{r}, \vec{\rho})
$$

and define the effective potential between $N\left(\vec{x}_{1}\right)$ and $N\left(\vec{x}_{2}\right)$ via the effective Schrödinger equation,

$$
\left[-\frac{1}{2 \mu_{r}} \nabla_{r}^{2}+V_{e f f}(\vec{r})\right] \phi(\vec{r})=E \phi(\vec{r})
$$

In this calculation, the DoF of $\vec{\rho}$ is integrated out beforehand, and thus the calculation cost is reduced by a factor of $\sim 1 / L^{3}$, compared to the straightforward calculation. Yet, the calculation remains quite expensive due to the large numbers of Wick contractions, and we use several techniques to reduce the calculation cost, e.g., we take advantage of symmetries (such as isospin symmetry), and we employ the non-relativistic limit for the source nucleon operator. Similar techniques are (independently) developed in the calculations of binding energies of triton/helium nuclei [प]].

As the $3 \mathrm{~N}$ system, we study the triton channel, $I=1 / 2, J^{P}=1 / 2^{+}$. Because the spectator nucleon is projected to the $\mathrm{S}$-wave, the possible quantum numbers between the (effective) $2 \mathrm{~N}$ are only ${ }^{2 S+1} L_{J}={ }^{1} S_{0},{ }^{3} S_{1},{ }^{3} D_{1}$, and we can obtain the effective $2 \mathrm{~N}$ potentials $V_{\text {eff }}(\vec{r})$ in parity-even channel, i.e., the central $V_{C, e f f}^{I=1, S=0}, V_{C, e f f}^{I=0, S=1}$ and the tensor $V_{T, e f f}^{I=0, S=1}$ potentials. We calculate all counterparts of these parity-even potentials in the genuine $2 \mathrm{~N}$ system, $V_{2 N}(\vec{r})$, and compare them with $V_{\text {eff }}(\vec{r})$ to extract the effect of the $3 \mathrm{~N}$ system. In one sense, $V_{e f f}(\vec{r})-V_{2 N}(\vec{r})$ can be considered to be the "finite density effect" in the $3 \mathrm{~N}$ system. Some of this effect are attributed to the genuine $2 \mathrm{~N}$ potential with the nontrivial $3 \mathrm{~N}$ correlation, and the others are originated by the genuine TNF. In this way, we can (indirectly) access the effect of TNF.

\section{Lattice setup and the results for the effective $2 \mathrm{~N}$ potential}

We employ $N_{f}=2$ dynamical configurations with mean field improved clover fermion and RG-improved gauge action generated by CP-PACS Collaboration [U]]. We use 598 configurations at $\beta=1.95$ with the lattice size of $L^{3} \times T=16^{3} \times 32$, which corresponds to $(2.5 \mathrm{fm})^{3}$ box in physical spacial size with the lattice spacing of $a^{-1}=1.269 \mathrm{GeV}$. We calculate at the hopping parameter of $u, d$ quarks $\kappa_{u d}=0.13750$, which corresponds to $m_{\pi}=1.13 \mathrm{GeV}, m_{N}=2.15 \mathrm{GeV}$. We use the wall quark source with Coulomb gauge fixing. In order to enhance the statistics, we perform the calculation for sources on 16 time slices for each configuration. 


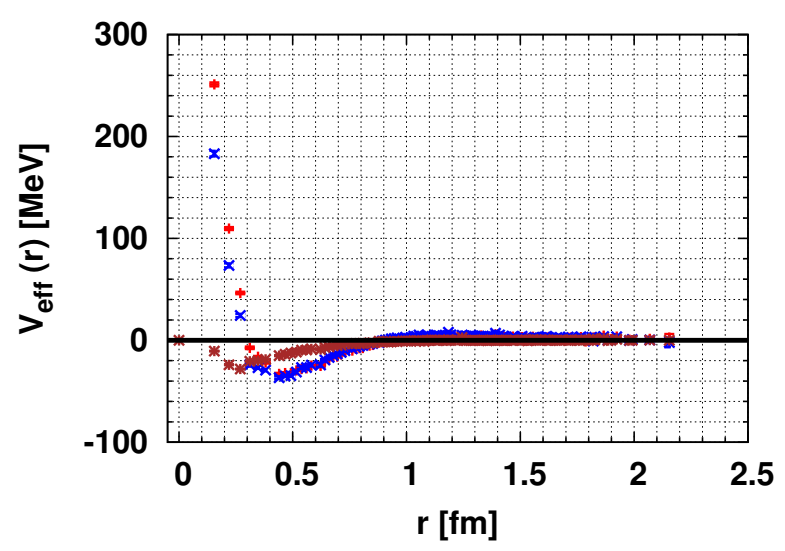

Figure 1: $\quad$ Effective $2 \mathrm{~N}$ potentials, where red, blue, brown points correspond to $V_{C, \text { eff }}^{I=1, S=0}$, $V_{C, e f f}^{I=0, S=1}, V_{T, e f f}^{I=0, S=1}$ potential, respectively.

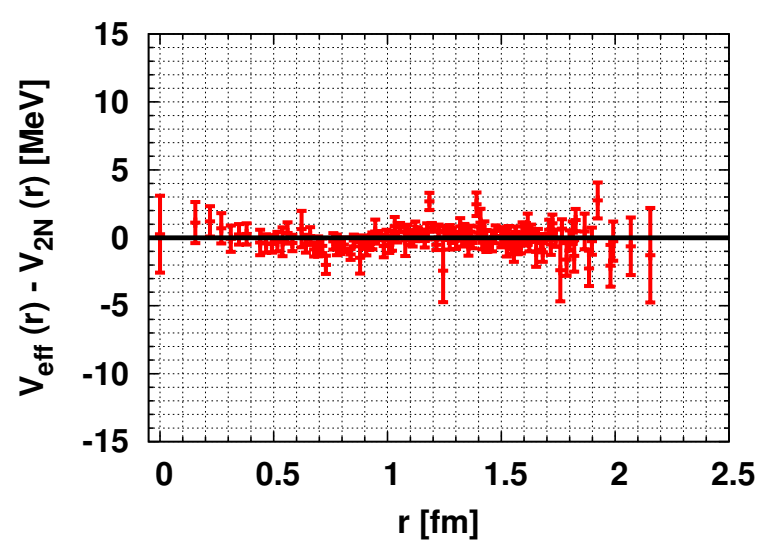

Figure 3: Same as Fig. 凤, but for $V_{C}^{I=0, S=1}$.

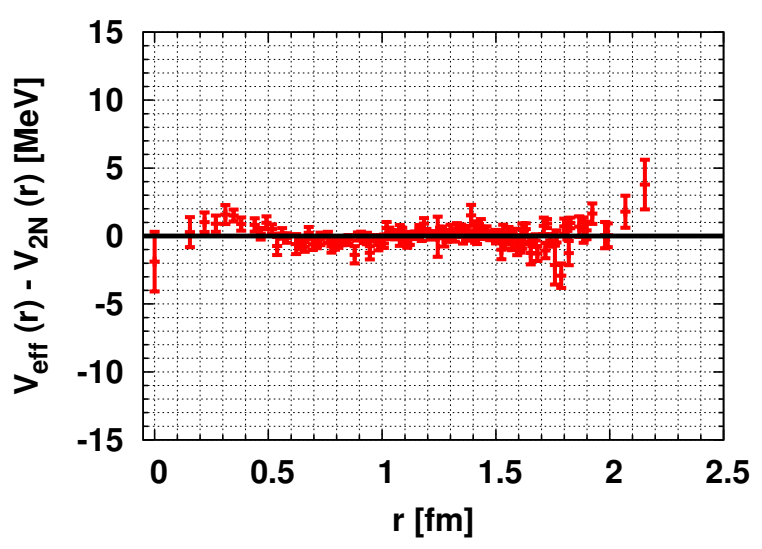

Figure 2: The difference between the effective $2 \mathrm{~N}$ and the genuine $2 \mathrm{~N}$ for $V_{C}^{I=1, S=0}$ potential.

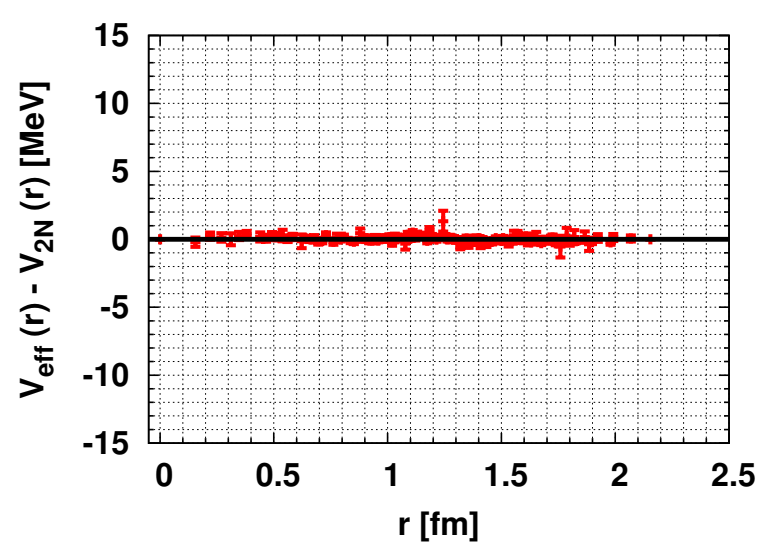

Figure 4: Same as Fig. 凤, but for $V_{T}^{I=0, S=1}$.

In Fig. [, we show preliminary results for $V_{\text {eff }}(r)$ in the triton channel at $t-t_{0}=8$. Here, the constant shift by energy is not included for the central potentials. What is noteworthy is that $V_{e f f}(r)$ are obtained with good precision. This is quite nontrivial, since the $\mathrm{S} / \mathrm{N}$ usually gets worse for more quarks in the system. In Figs. [1], see that the discrepancy is consistent with zero within error-bar. In particular, the tensor potential is best constrained within several $\mathrm{MeV}$ statistical error, and there is no indication of the TNF effect.

One of the possible explanations why the TNF effect is not observed is that the TNF is suppressed at heavy quark mass. Actually, pion exchange is expected to be strongly suppressed with $m_{\pi}=1.13 \mathrm{GeV}$ in this calculation. Therefore, we investigate the quark mass dependence using the configurations with smaller quark masses, generated by PACS-CS Collaboration [12]: $N_{f}=2+1$ nonperturbatively $\mathscr{O}(a)$ improved clover fermion, $V=32^{3} \times 64, \beta=1.90, a^{-1}=2.18 \mathrm{GeV}$. We use 399 configurations at $\kappa_{u d}=0.13700, \kappa_{s}=0.13640\left(m_{\pi}=0.70 \mathrm{GeV}, m_{N}=1.58 \mathrm{GeV}\right)$ and 400 configurations at $\kappa_{u d}=0.13727, \kappa_{s}=0.13640\left(m_{\pi}=0.57 \mathrm{GeV}, m_{N}=1.41 \mathrm{GeV}\right)$. For both quark mass setups, we perform 4 source time slice measurements for each configuration.

We obtain basically similar results to previous results: For all potentials at either quark mass, the differences between $V_{e f f}(r)$ and $V_{2 N}(r)$ are found to be consistent with zero within error-bar. In particular, the statistical error of $V_{e f f}(r)-V_{2 N}(r)$ in tensor potential remains less than $5 \mathrm{MeV}$ even 
for the lightest quark mass. This may indicate the necessity to decrease the quark mass further.

Another possible explanation is that the TNF effect is obscured by the summation over the location of the spectator nucleon. In fact, while the TNF effect is expected to be enhanced when all three nucleons are close to each other, such 3D-spacial configurations make small contributions in the spectator summation. In order to assess this possibility, we study the $3 \mathrm{~N}$ system with (at closely) fixed 3D-configuration, which is the subject of the next section.

\section{Formulation for the fixed 3D-configuration with the linear setup}

We consider Eq. (2.2) with fixed 3D-configuration of $\vec{r}, \vec{\rho}$. The advantage of this method is that the subtraction procedure of the genuine $2 \mathrm{~N}$ potentials $V_{2 N}$ becomes clearer in principle, and thus the TNF can be extracted directly. Furthermore, by choosing small $|\vec{r}|,|\vec{\rho}|$ configurations, the TNF effect is expected to be enhanced. The disadvantage of this calculation is the calculation cost: It is more expensive by a factor of $\mathscr{O}(10)-\mathscr{O}\left(10^{2}\right)$, compared to the effective $2 \mathrm{~N}$ potential study. Therefore, we restrict the calculation to limited 3D-configurations. In addition, efforts are taken to speed up the calculation code. As the fixed 3D-configuration, we take the linear setup with $\vec{\rho}=\overrightarrow{0}$. Just for the sake of convenience, we redefine as $\vec{r} \rightarrow 2 \vec{r}$ hereafter. In other words, three nucleons are aligned linearly with equal spacings of $r=|\vec{r}|$ in this linear setup.

The advantage of the linear setup is it's simplicity. Because of $\vec{\rho}=\overrightarrow{0}$, the third nucleon is attached to $(1,2)$-nucleon pair with only $\mathrm{S}$-wave. Considering the total $3 \mathrm{~N}$ quantum numbers of $I=1 / 2, J^{P}=1 / 2^{+}$, the wave function can be completely spanned by only three bases, which can be labeled by the quantum numbers of $(1,2)$-pair as ${ }^{1} S_{0},{ }^{3} S_{1},{ }^{3} D_{1}$. Therefore, the Schrödinger equation can be simplified to the $3 \times 3$ coupled channel equations with the bases of $\psi_{1} S_{0}, \psi_{3} S_{1}$, $\psi^{3} D_{1}$. The reduction of the dimension of bases is expected to improve the $\mathrm{S} / \mathrm{N}$ as well.

Unfortunately, even by the calculation of the fixed 3D-configuration (including the linear setup), the subtraction of $V_{2 N}$ remains nontrivial. As was noted in Sec. 凹, the parity-odd potentials are not available in lattice QCD at this moment, and we cannot subtract them unambiguously. Note that although the total parity of the $3 \mathrm{~N}$ system can be projected, a $2 \mathrm{~N}$-pair could be either of positive or negative parity. The familiar procedure of partial wave expansion cannot be performed here, since we can calculate only limited 3D-configurations due to the huge calculation cost.

In the effort to overcome this issue, we find that the following channel in the triton is useful,

$$
\psi_{S} \equiv \frac{1}{\sqrt{6}}\left[-p_{\uparrow} n_{\uparrow} n_{\downarrow}+p_{\uparrow} n_{\downarrow} n_{\uparrow}-n_{\uparrow} n_{\downarrow} p_{\uparrow}+n_{\downarrow} n_{\uparrow} p_{\uparrow}+n_{\uparrow} p_{\uparrow} n_{\downarrow}-n_{\downarrow} p_{\uparrow} n_{\uparrow}\right] .
$$

This wave function itself has been well known, but the point here is that it is anti-symmetric in spin/isospin spaces for any $2 \mathrm{~N}$-pair. Combined with the Pauli-principle, it is automatically guaranteed that any $2 \mathrm{~N}$-pair couples with even parity only. Therefore, we can extract the TNF unambiguously in this channel, without the information of parity-odd $2 \mathrm{~N}$ potentials. Note that no assumption on the choice of 3D-configuration is imposed in this argument, and we can take advantage of this feature for the future TNF calculations with 3D-configurations other than the linear setup.

Coming back to the linear setup, we examine the explicit form of the potential matrix of $V_{2 N}$. At the leading order of the velocity expansion, $V_{2 N}$ can be written in terms of center $V_{C}^{I S}$ and tensor $V_{T}^{I S}$ potentials with isospin $I$ and $\operatorname{spin} S, V_{C}^{00}, V_{C}^{10}, V_{C}^{01}, V_{C}^{11}, V_{T}^{01}, V_{T}^{11}$, where the label " $2 N$ " is omitted for simplicity. Explicit calculation gives us 


$$
V_{2 N}=\left(\begin{array}{c|c|c}
+V_{C}^{10}(r)+V_{C}^{01}(r) & +\frac{1}{2} V_{C}^{10}(r)-\frac{1}{2} V_{C}^{01}(r) & -2 V_{T}^{01}(r) \\
+\frac{1}{2} V_{C}^{10}(2 r)+\frac{1}{2} V_{C}^{01}(2 r) & -\frac{1}{2} V_{C}^{10}(2 r)+\frac{1}{2} V_{C}^{01}(2 r) & +2 V_{T}^{01}(2 r) \\
\hline+\frac{1}{2} V_{C}^{10}(r)-\frac{1}{2} V_{C}^{01}(r) & +\frac{3}{4} V_{C}^{00}(r)+\frac{1}{4} V_{C}^{10}(r)+\frac{1}{4} V_{C}^{01}(r)+\frac{3}{4} V_{C}^{11}(r) & +V_{T}^{01}(r)-3 V_{T}^{11}(r) \\
-\frac{1}{2} V_{C}^{10}(2 r)+\frac{1}{2} V_{C}^{01}(2 r) & +\frac{1}{2} V_{C}^{10}(2 r)+\frac{1}{2} V_{C}^{01}(2 r) & +2 V_{T}^{01}(2 r) \\
\hline-2 V_{T}^{01}(r) & +V_{T}^{01}(r)-3 V_{T}^{11}(r) & +\frac{1}{2} V_{C}^{01}(r)+\frac{3}{2} V_{C}^{11}(r)-V_{T}^{01}(r)-3 V_{T}^{11}(r) \\
+2 V_{T}^{01}(2 r) & +2 V_{T}^{01}(2 r) & +V_{C}^{01}(2 r)-2 V_{T}^{01}(2 r)
\end{array}\right) \text { (4.2) }
$$

Here, we span the spaces with the rotated bases given by $\left(\psi_{S}, \psi_{M}, \psi_{3} D_{1}\right)^{T}$, where $\psi_{S}$ in Eq. (4.CI) is shown to be $\psi_{S}=\frac{1}{\sqrt{2}}\left(-\psi_{S^{1}}+\psi_{S^{3}}\right)$, and $\psi_{M} \equiv \frac{1}{\sqrt{2}}\left(+\psi_{1} S_{0}+\psi_{S^{3}}\right)$. Note that neither of parity-odd $2 \mathrm{~N}$ potentials, $V_{C}^{00}, V_{C}^{11}, V_{T}^{11}$, appear in the first row in Eq. (4.2), as was discussed previously.

\section{The lattice $Q C D$ results for the linear setup}

We employ the CP-PACS $N_{f}=2$ clover fermion configurations [W], which are used for the effective $2 \mathrm{~N}$ potential study in Sec. [1]. 598 configurations are used with 16 source time slice measurements for each configuration. We perform the calculation with the linear setup at 7 physical points of the distance $r$. As is explicitly shown in Eq. (4.2), we have only one channel which is free from parity-odd $2 \mathrm{~N}$ potentials. Correspondingly, we can determine one type of TNF. In this proceeding, we consider the scalar/isoscalar type TNF. In fact, in the Urbana IX model [四], which is an oftenused phenomenological TNF, TNF consists of two parts, one is the two-pion exchange TNF (which has spin/isospin dependencies), and the other is phenomenologically introduced scalar/isoscalar repulsive TNF. Since the two-pion exchange TNF is expected to be suppressed in the current lattice setup of $m_{\pi}=1.13 \mathrm{GeV}$, it is reasonable to assume the scalar/isoscalar nature for TNF here.

In Fig. [, we plot each wave function of $\psi_{S}, \psi_{M}, \psi_{3^{3} D_{1}}$ in the triton channel at $t-t_{0}=8$. We observe that $\psi_{S}$ dominates the wave function. This is because $\psi_{S}$ contains the component for which all three nucleons are in S-wave. We emphasize that the observation of good S/N for the wave function is much more nontrivial than the effective $2 \mathrm{~N}$ study, because the practical statistical sampling number becomes much smaller by fixing the 3D-configuration.

By subtracting the $V_{2 N}$ in Eq. (4.2) from the total potentials in the $3 \mathrm{~N}$ system, we determine the TNF. In Fig. G, we plot the preliminary results for the scalar/isoscalar TNF. Here, the $r$-independent shift by energies is not included, and thus about $\mathscr{O}(10) \mathrm{MeV}$ systematic error is understood. There are various physical implications in Fig. 6 . At the long distance region of $r$, the TNF is small as is expected. At the short distance region, we observe the indication of repulsive TNF. Recalling that the repulsive short-range TNF is phenomenologically required to explain the saturation density of nuclear matter, etc., this is very encouraging result. Of course, we note that further study is necessary to confirm this result, e.g., the study of the ground state saturation, the evaluation of the constant shift by energies, the examination of the discretization error.

\section{Summary}

We have studied the three nucleon force (TNF) in the triton channel in lattice QCD, developing two different methods. In the first method, effective $2 \mathrm{~N}$ potentials have been studied using $N_{f}=2$ 


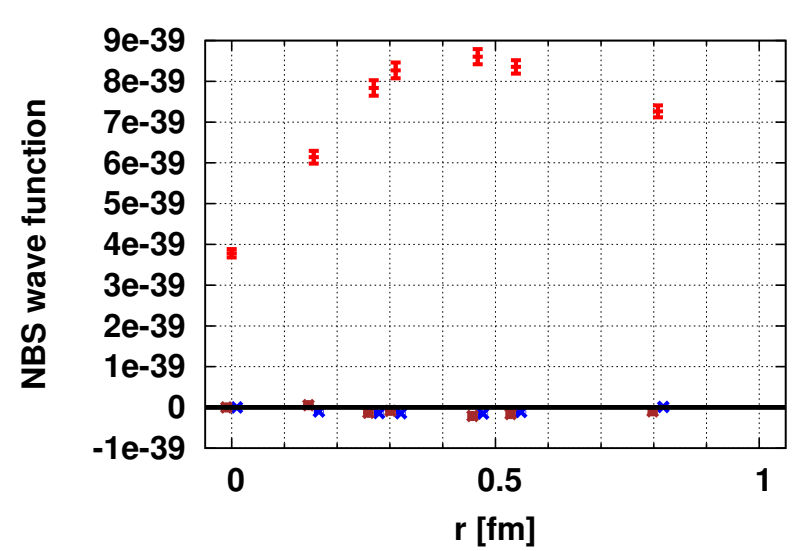

Figure 5: The wave function with linear setup in the triton channel. Red, blue, brown points correspond to $\psi_{S}, \psi_{M}, \psi_{3} D_{1}$, respectively.

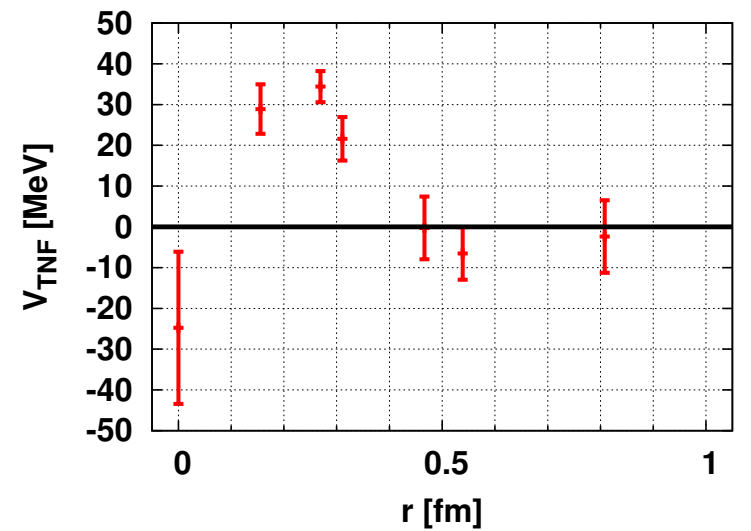

Figure 6: The scalar/isoscalar TNF in the triton channel, plotted against the distance $r$ in the linear setup.

clover fermion at $m_{\pi}=1.13 \mathrm{GeV}$, and $N_{f}=2+1$ clover fermion at $m_{\pi}=0.70,0.57 \mathrm{GeV}$. The effective $2 \mathrm{~N}$ potentials have been found to be consistent with genuine $2 \mathrm{~N}$ potentials within error. In the second method, we have fixed the 3D-configuration of three nucleons. In particular, we have established the general procedure which can identify the TNF without the information of parityodd $2 \mathrm{~N}$ potentials. The calculation have been performed with $N_{f}=2$ clover fermion at $m_{\pi}=1.13$ $\mathrm{GeV}$ with the linear setup for the 3D-configuration, and the indication of repulsive TNF at short distances have been obtained. Further work to confirm the results is currently underway.

We thank CP-PACS and PACS-CS Collaborations and ILDG/JLDG [ए3] for providing the configurations. TD is supported in part by Grant-in-Aid for JSPS Fellows 21.5985. This research is supported in part by Grant-in-Aid for Scientific Research on Innovative Areas (No.2004:20105001, 20105003) and the Large Scale Simulation Program No.09-23 (FY2009) of KEK. The numerical calculations have been performed on T2K at University of Tsukuba and Blue Gene/L at KEK.

\section{References}

[1] T. Otsuka et al., Phys. Rev. Lett. 105, 032501 (2010); J.D. Holt et al., arXiv:1009.5984 [nucl-th]

[2] K. Sekiguchi et al., Phys. Rev. C 79, 054008 (2009) and references therein.

[3] J. Fujita and H. Miyazawa, Prog. Theor. Phys. 17, 360 (1957).

[4] B.S. Pudliner, V.R. Pandharipande, J. Carlson and R.B. Wiringa, Phys. Rev. Lett. 74, 4396 (1995).

[5] U. van Kolck, Phys. Rev. C 49, 2932 (1994).

[6] N. Ishii, S. Aoki and T. Hatsuda, Phys. Rev. Lett 99, 022001 (2007).

[7] S. Aoki, T. Hatsuda and N. Ishii, Prog. Theor. Phys. 123, 89 (2010).

[8] H. Nemura, N. Ishii, S. Aoki and T. Hatsuda, Phys. Lett. B 673, 136 (2009).

[9] T. Inoue et al. (HAL QCD Collab.), arXiv:1007.3559 [hep-lat], in press in Prog. Theor. Phys.

[10] T. Yamazaki, Y. Kuramashi and A. Ukawa, Phys. Rev. D 81, 111504 (2010).

[11] S. Aoki et al. (CP-PACS Collab.), Phys. Rev. D 65, 054505 (2002) [E: D 67,059901 (2003)]

[12] S. Aoki et al. (PACS-CS Collab.), Phys. Rev. D 79, 034503 (2009).

[13] "http://www.lqcd.org/ildg" / "http://www.jldg.org" 